\title{
Comparison between Shear Strength of Dry Sand Measured in CSS Device using Wire-reinforced Membranes and Stacked Rings
}

\author{
Wing Shun Kwan ${ }^{1}$, S.M. ASCE, P.E. and Chadi El Mohtar², A.M.ASCE, Ph.D. \\ ${ }^{1}$ Graduate Student, Civil, Architectural and Environmental Engineering, The \\ University of Texas at Austin, ECJ 9.227, 301 E. Dean Keeton Stop C1792 \\ Austin, TX $78712-0280$ \\ ${ }^{2}$ Assistent Professor, Civil, Architectural and Environmental Engineering, The \\ University of Texas at Austin, ECJ 9.227B, 301 E. Dean Keeton Stop C1792 \\ Austin, TX $78712-0280$
}

\begin{abstract}
The cyclic simple shear test (CSS) is widely regarded as the preferred element test for liquefaction because it best simulates vertically propagating shear waves. The CSS test provides reliable, high-quality laboratory data for model development and calibration in Geotechnical Earthquake Engineering design. Direct simple shear (DSS) tests can be performed by using the same apparatus to measure the shear strength and define the failure envelope. The consolidation and shearing are performed under $\mathrm{K}_{0}$ conditions. The $\mathrm{K}_{0}$ conditions are maintained through lateral confinement that prevents lateral deformations. Wire-reinforced membranes (WR) and stacked rings (SR) have been the two major techniques used to achieve the no lateral strain condition. The motivation for this work was to study the effect of using a WR versus SR on the stress-strain behavior and shear strength of clean sand. The paper presents results from DSS tests performed on Monterey \#0/30 and Washed Mortar sands. The tests were performed under a constant vertical stress instead of constant volume to monitor effect of the confinement systems on the contractive and dilative response of the sand samples. The results show that there is general agreement between the measured shear strength and volumetric response with SR and WR confinement systems.
\end{abstract}

\section{INTRODUCTION}

Liquefaction of saturated loose sand has been the topic of extensive laboratory research over the past 40 years after the two devastating earthquakes of 1964 in Niigata, Japan and Alaska. Cyclic triaxial (CTX), torsional, and simple shear tests are used today for soil element-level testing. Triaxial and simple shear apparatuses are the most popular and commonly used. The CSS test provides better simulations of in-situ stresses for seismic hazard evaluations than the CTX because it is capable of reproducing the seismic loading conditions (Boulanger et al. 1993). The 'simple shear' mechanism allows the principal stress axes to rotate smoothly during cyclic loading, 
while the principal axes rotate 90 degrees instantaneously in CTX. CSS also allows for $\mathrm{K}_{0}$ consolidation, which prevents any lateral strain and maintains constant volume during undrained loading. Instead of applying cell pressure through a latex membrane to achieve $\mathrm{K}_{0}$ conditions, lateral confinement in simple shear testing is maintained through the use of NGI-type wire-reinforced latex membranes or stacked rings to provide lateral constrain while applying a vertical stress.

CSS testing has been in practice for a few decades, and different designs have been used. WR is commonly used in academic liquefaction testing (Bjerrum and Landva 1966, Boulanger et al. 1993, Kammerer et al. 2002,), but is not the only option. SR (Ishihara and Yamazaki 1980) is also available and attractively simple in sample preparation. In fact, SR has become more popular in engineering practices over the past ten to twenty years after automated simple shear apparatuses became commercially available. SR is usually sold along with the commercial equipment for its cost effectiveness and durability.

The stress state in the simple shear apparatus can be determined by conducting Direct Simple Shear (DSS) tests. Despite that a plane strain condition is well maintained at the consolidation stage, Roscoe (1953) has proven the shear stress at the specimen top and bottom could be non-uniform during the shearing stage. The normal stress could also be non-uniformly distributed (Wood et al. 1980). The specimen's circular shape prevents direct measurements of the forces on the confinement side walls, and therefore it is almost impossible to directly quantify the lateral confinements from the WR or SR. However, the effect of WR versus SR can be concluded from the stress-strain response under DSS tests.

Baxter et al. (2010) showed that the two confinement systems give comparable test results on cohesive soils in DSS tests sheared at constant volume. This paper presents DSS test results on clean dry sand to study their stress-strain behavior and shear strength under the two different confinement methods when under a constant vertical load. While this method is a modification form the traditional way of running DSS at a constant volume, it better simulates the way the CSS is run when pore pressures are measured (as compared to at a constant volume). Whether running the test under a constant normal load or a constant volume, the results will reflect any bias introduced by the two different confining methods.

\section{TESTING METHODS}

A laboratory-testing program was performed at the University of Texas at Austin using the Geotechnical Consulting and Testing System (GCTS) manufactured Cyclic Simple Shear apparatus. The system uses a closed loop, electro-hydraulically actuated, servo valve that controls shearing in the horizontal direction at the bottom platen under load or displacement controls. A total of 40 DSS tests were performed on two types of dry sands, Monterey\#0/30 and Washed Mortar, at two different relative densities. The sand properties are presented in Table 1 and the particle size gradation is shown in Figure 2. According to USCS, both sands are classified as a uniform sand (SP). 


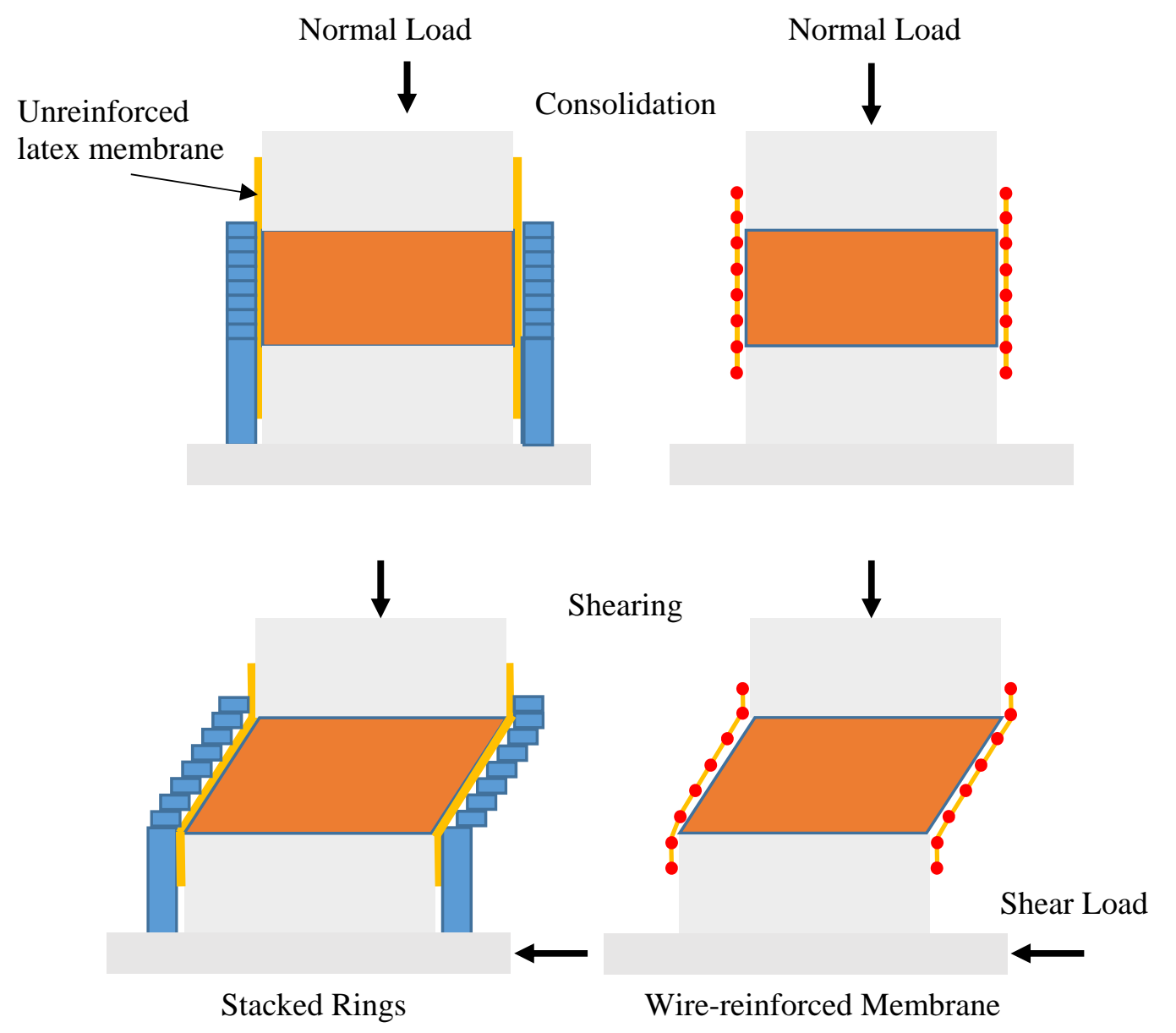

FIG. 1. Setup of Stacked Rings and Wire-reinforced during consolidation and shearing

Table 1. Soil Properties of Tested Sand

\begin{tabular}{|c|c|c|c|c|c|}
\hline Sand Type & $\mathbf{e}_{\min }$ & $\mathbf{e}_{\max }$ & S.G. & $\mathbf{C}_{\mathbf{u}}$ & $\mathbf{C}_{\mathbf{c}}$ \\
\hline Monterey \#0/30 & 0.57 & 0.85 & 2.64 & 1.6 & 2.1 \\
\hline Washed Mortar & 0.56 & 0.84 & 2.65 & 2.6 & 4.1 \\
\hline
\end{tabular}

A rectangular aluminum bracket was fabricated and installed above the top platen to minimize any tilting at the top platen during shear. Two linear variable differential transformers (LVDT) were used to measure the horizontal and vertical displacements (Figure 3). The vertical LVDT was used to monitor any volumetric change from the soil specimen or tilting from the top platen during shear; the shear LVDT was setup horizontally to measure relative movement of the bottom platen relative to the top one. All specimens were sheared at a shear strain rate of $0.25 \%$ per minute. 


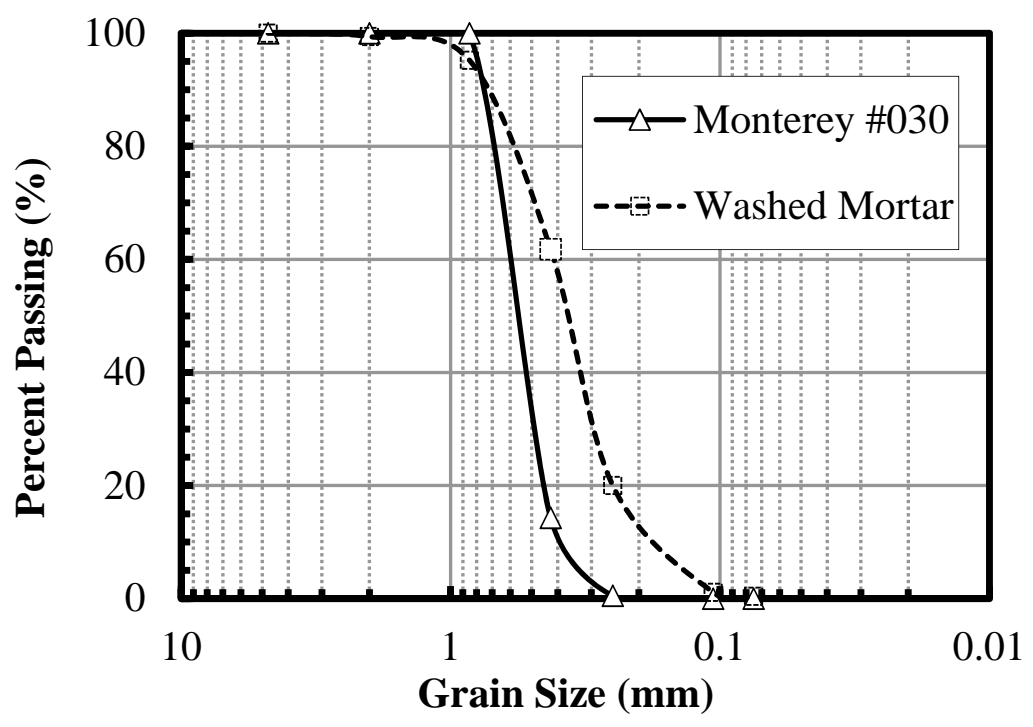

FIG. 2. Grain Size disturbution of tested soils

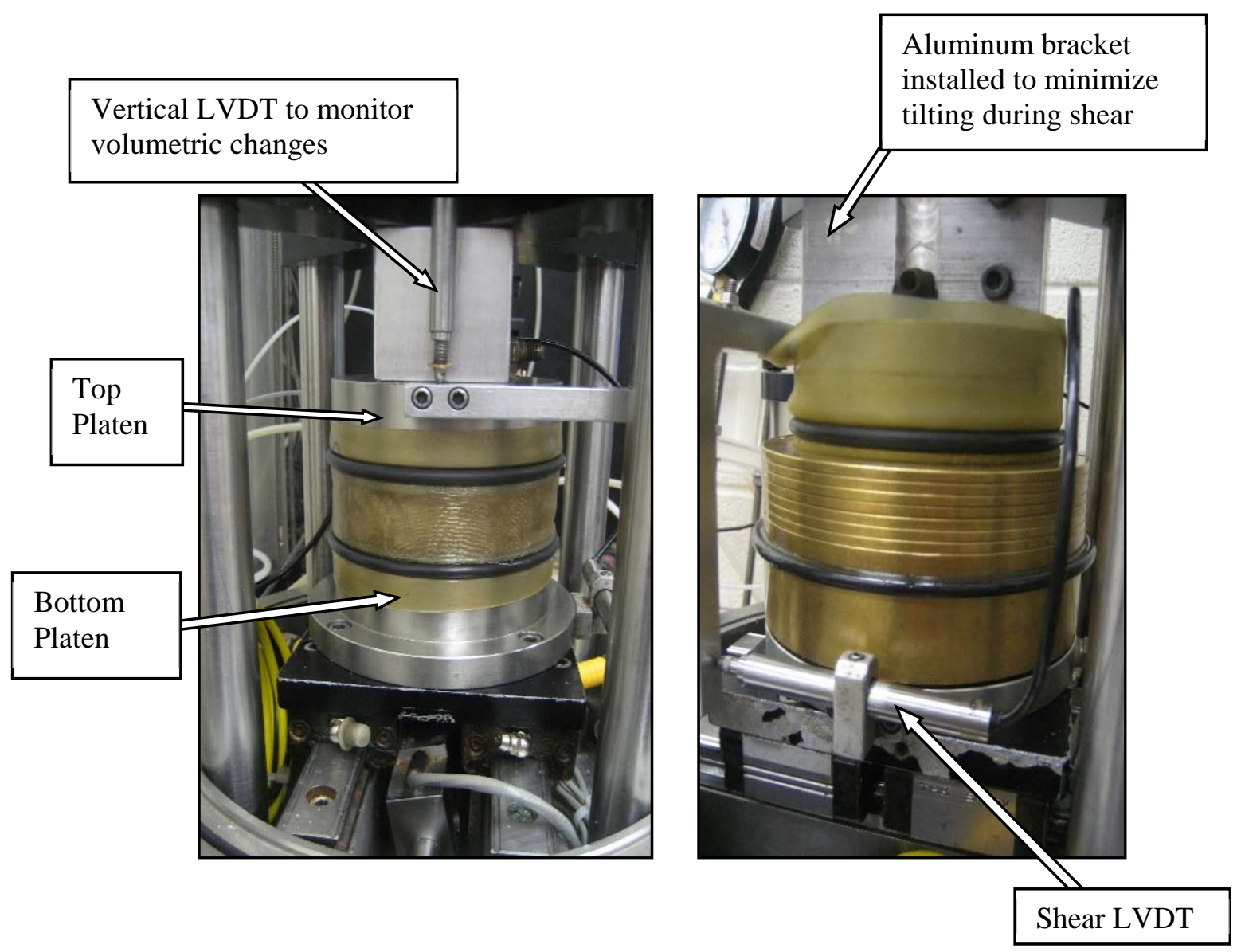

FIG. 3. Setup of Specimens under Confinements of Wire-Reinforced Membrane (left) and Stacked Rings (right) 


\section{SAMPLE PREPARATION}

All tested specimens were 1" high and 4" in diameter. A standard split mold was used to stretch the wire-reinforced membrane and ensure a circular cross-section before placing the sand in. The wire-reinforced membrane used was manufactured by NGI, moderate stiffness $(\mathrm{C}=1.0)$. For the stacked rings, a special split mold was designed and manufactured for this study. The new split mold surrounds the stacked rings as well as the latex membrane; therefore, when vacuum is applied, the latex membrane is pulled in contact with the stacked rings to avoid any gaps between the two. Lubricant oil was applied between the stack rings to minimize friction. Once the vacuum was applied, the sand specimens were reconstituted following the dry funnel deposition method. The spout of a funnel filled with sand was placed at the bottom platen and then it was raised slowly while going around the area in circles. The free drop height of the sand determines the relative density of the soil specimen; loose specimens were created with a drop height of zero while a higher density was reached by a greater drop height followed by gently tapping on the split mold in a symmetrical pattern. After leveling the top of the sand, the top platen was placed on top of the sand and a seating vertical stress was applied to the specimen. The split mold was then removed and the specimen was consolidated to a vertical stresses of 25, 50, 75, 100 and $150 \mathrm{kPa}$ before shearing.

In addition to soil specimens, water specimens were created to investigate the shear resistance provided by the two confining systems. Water is assumed to have no shear resistance, and therefore the measured resistance during shearing is contributed by the confining system and friction within the equipment. Figure 4 shows the water tests results. At a small strain $(<1 \%)$, the resistances from SR and WR both increased quickly to $0.5 \mathrm{kPa}$, and the measured resistances are very close to each other. In both systems there is a sharp increase in shear stress right after shearing starts, followed by a linear increase at a flatter slope up to a shear strain of $25 \%$. Since the magnitudes of the systems' resistance were relatively small, a simple linear model with a y-intercept was used to correct the measured shear stresses during later tests. Both systems had a very similar $y$-intercept (a measure of the friction in the loading mechanism rather than resistance of the confinement); however, the increase in resistance of the SR with increasing shear strain is slightly higher than that of the WR after the initial spike.

\section{DSS TEST RESULTS AND DATA COMPARISON}

Figures 5 and 6 present the corrected shear resistance and change in specimen height (positive for compression and negative for dilation) during shear for Monterey and Washed Mortar sands, respectively, using the two different confinement systems. Table 2 summarized the range of relative densities for the tests performed. Overall, the results from both confinement methods were consistent with Monterey \#0/30 (more uniform sand), showing less discrepancy than Washed Mortar sand.

Comparisons of stress strain behaviors could depend on soil type, density, and stress level. Greater discrepancy is observed in dense Washed Mortar sands at higher vertical confining stresses. Tests confined with SR showed a stiffer and more dilative response than WR when dense sand was sheared under vertical confining stresses of 
100 and $150 \mathrm{kPa}$, as shown in Figure 6. SR provided a stiffer confinement than WR. The large particles were prone to dilate in SR than WR. For Monterey \#0/30 sand, specimens tested with WR had a stiffer response than SR in all tests, with the exception of one test performed on loose density at $150 \mathrm{kPa}$ vertical stress. The two different confinement systems provide overall conformity in stress-strain relationship and maximum shear stress.

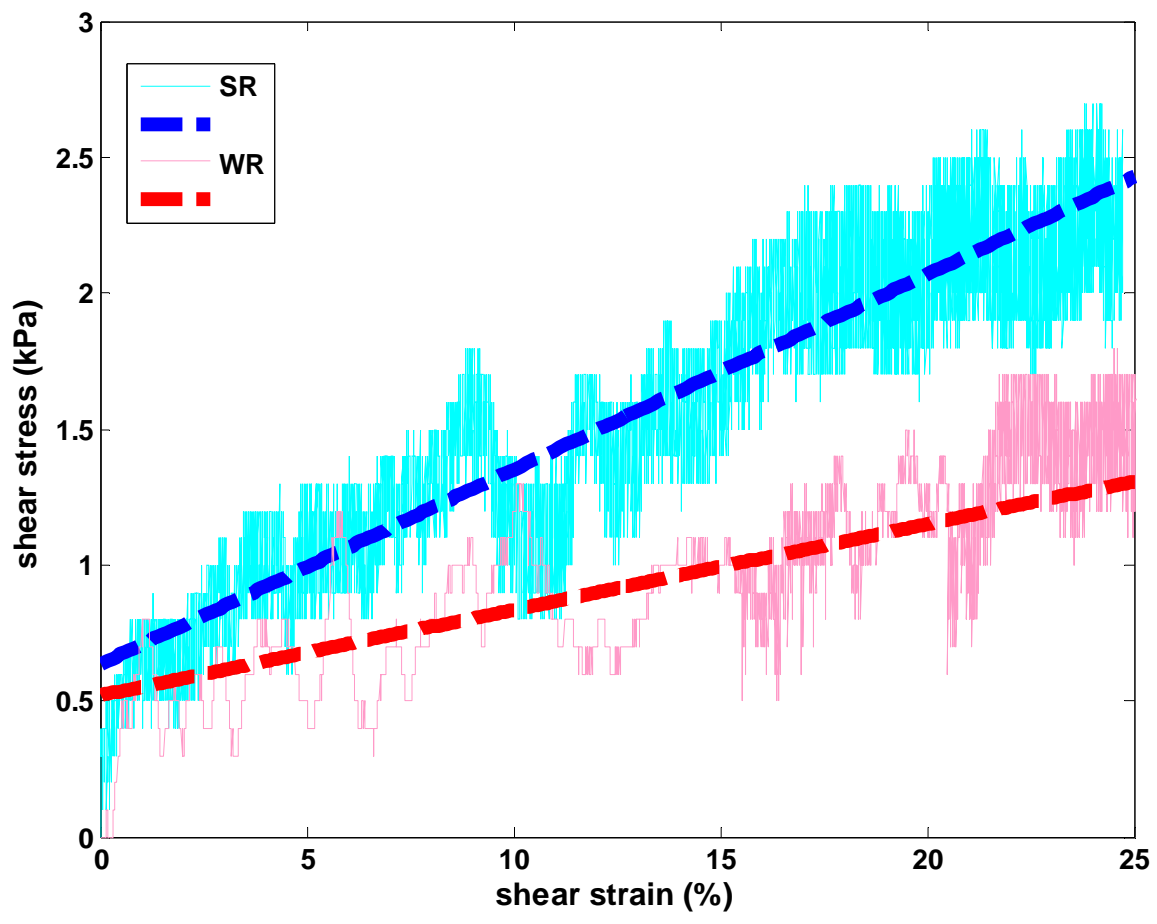

FIG. 4. Stress-Strain plot for water specimens under different confining systems

Table 2. Range of relative densities for all tests

\begin{tabular}{|c|c|c|}
\hline Sand Type & Loose & Dense \\
\hline Monterey \#0/30 & $28 \%-44 \%$ & $66 \%-80 \%$ \\
\hline Washed Mortar & $23 \%-46 \%$ & $69 \%-82 \%$ \\
\hline
\end{tabular}

The volumetric change during shear can be represented by the change in specimen height since the confining systems restrict radial displacement. In general, the dense specimens tend to dilate and loose specimens tend to compress as indicated in Figures 5 and 6. However, critical state (constant volume) was not reached at large strain levels for most of the tests. This can be explained in part by non-uniform stress distributions and because the confining systems might have some flexibility (Saada 1983). At higher vertical stresses both sands showed a decrease in the dilative behavior. For tests on Monterey \#0/30 sand, the amount of compression is greater in tests with SR than WR in general, but it is not valid in tests with Washed Mortar sand. 
Monterey\#0/30 (Dense)
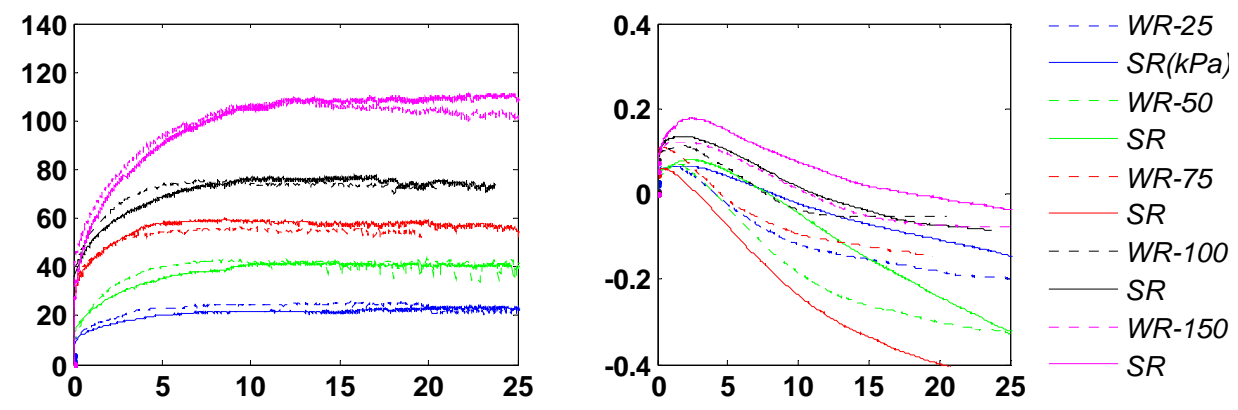

Monterey\#0/30 (Loose)
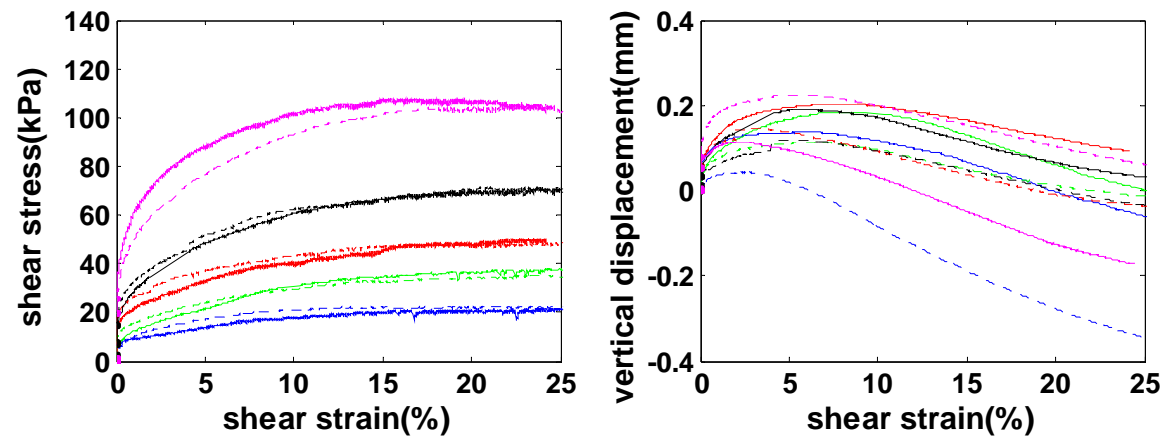

FIG. 5. Shear stress and vertical displacement verses shear strain for Monterey \#0/30 sand
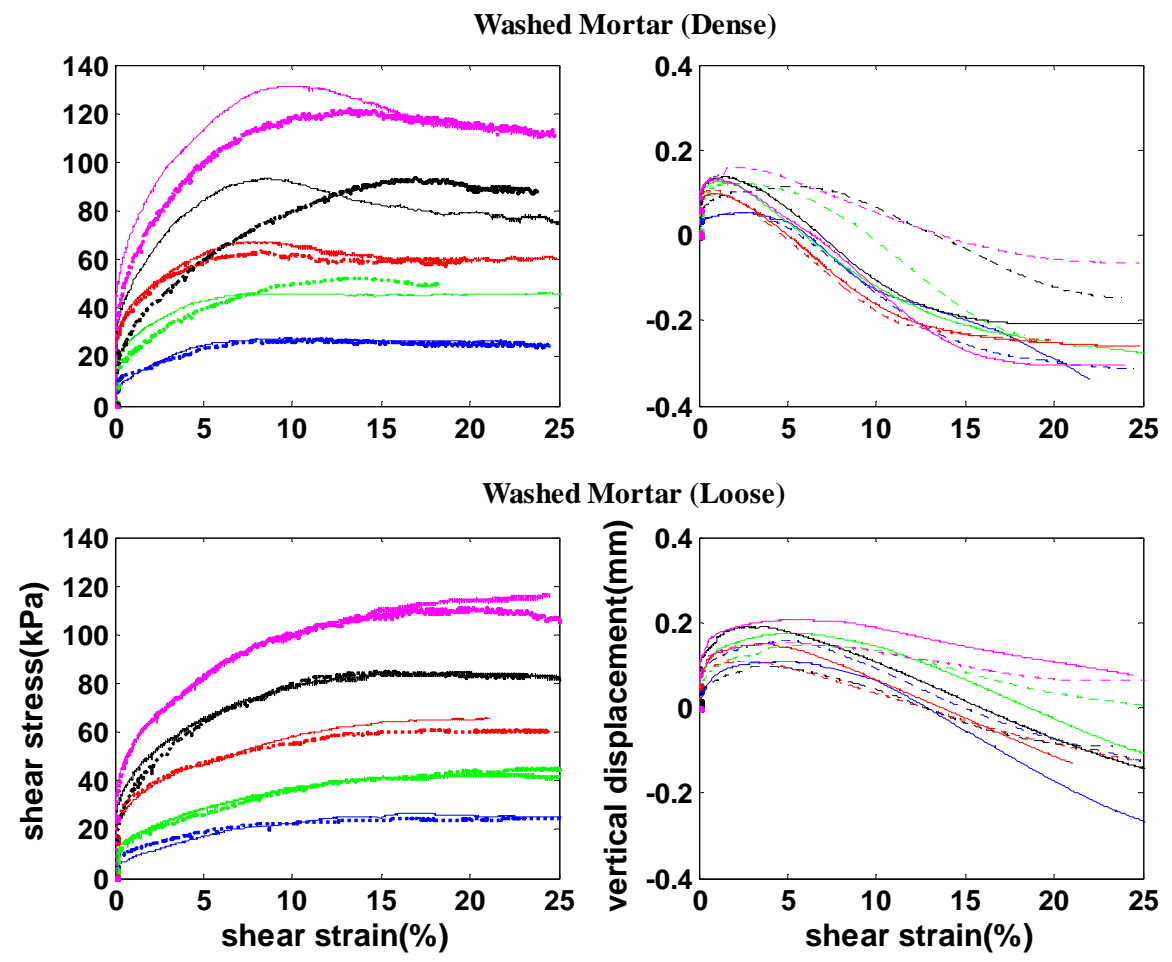

FIG. 6. Shear stress and vertical displacement verses shear strain for Washed Mortar Sand 
The strength envelops defined by the maximum shear stress and corresponding calculated friction angles $\left(\phi_{\max }^{\prime}\right)$ are shown on Fig 7 . Table 3 summarizes the recorded friction angles for all four cases. The difference between WR and SR is less than one degree in terms of friction angles, which proves that the two different confinement methods agree. The results also show that SR envelopes are very close or slightly higher than WR envelopes. Note that these results were corrected for the bias due to the equipment and confining friction mechanism by subtracting the recorded shear stresses in the water tests. The high friction angle recorded in the SR tests can be a result of slightly higher lateral confinement provided by the SR as compared to the WR.
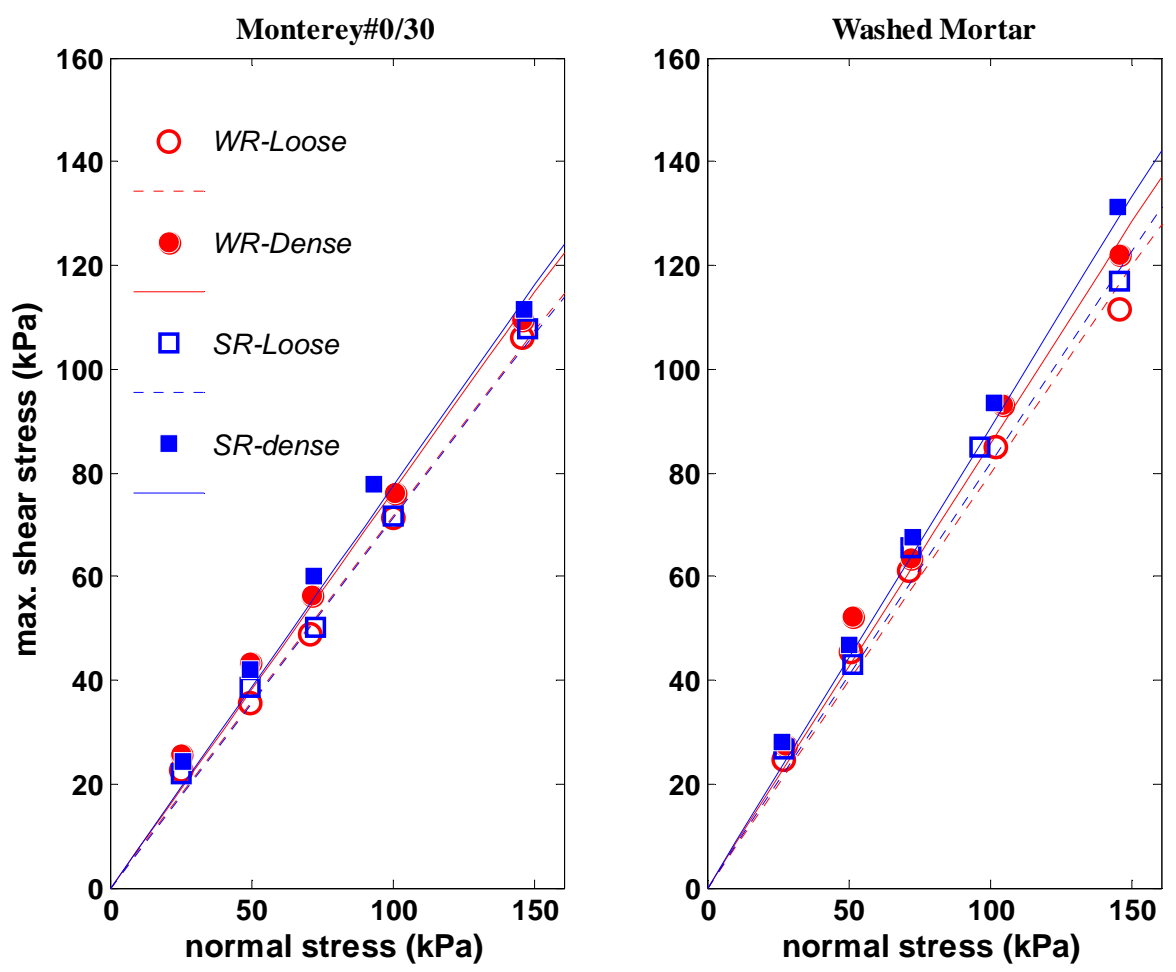

FIG. 7. Soil strength envelops from DSS tests

Table 3. Summary of friction angles from DSS tests

\begin{tabular}{|c|c|c|c|}
\hline Sand & Confinement Method & $\mathrm{D}_{\mathrm{r}}(\%)$ & $\phi^{\prime}$ (deg) \\
\hline \multirow{4}{*}{ Monterey \#0/30 } & \multirow{2}{*}{ WR } & $30-44$ & 35.6 \\
\hline & & $67-80$ & 37.4 \\
\hline & \multirow{2}{*}{ SR } & $28-42$ & 35.5 \\
\hline & & $66-73$ & 37.4 \\
\hline \multirow{4}{*}{ Washed Mortar } & \multirow{2}{*}{ WR } & $23-46$ & 38.6 \\
\hline & & $69-82$ & 40.6 \\
\hline & \multirow{2}{*}{ SR } & $23-37$ & 39.3 \\
\hline & & $70-77$ & 41.6 \\
\hline
\end{tabular}




\section{CONCLUSIONS}

This paper presented a comparison between the two popular lateral confining systems, wire-reinforced membrane and stacked rings, in CSS testing. DSS tests with the two systems were performed in a CSS apparatus to investigate the differences. The tests were performed under a constant vertical load rather than a constant volume to try to identify the effectiveness of the two confinement systems in maintaining the contractive versus dilative behavior. The results did not show a consistent bias in one of the systems versus another in terms of dilative versus contractive behavior.

Water tests were performed to measure the equipment and confining system's inherent resistance and correct for it. The results show SR gives a slightly higher resistance than WR. The DSS testing program shows comparable test results from the two confinement systems. Minor discrepancies were found between the two confining systems in terms of measured internal friction angle. These differences are dependent on soil gradation, density, and stress level. However, the friction angles measured with both systems were within one degree, which is considered to be comparable.

\section{ACKNOWLEDGEMENTS}

The authors would like to expression their appreciation to the Civil, Architectural and Environmental Engineering Department for supporting the first author during the period of performing these tests. The authors would like to acknowledge the efforts of Ms. Patricia Bennett, an undergraduate student at The University of Texas at Austin, for her contributions to this work.

\section{REFERENCES}

Baxter, B., Ochoa-Lavergne and R. Hankour (2010). "DSS Test Results Using WireReinforced Membranes and Stacked Rings." GeoFlorida 2010: Advances in Analysis, Modeling \& DesignFlorida, 600-607.

Bjerrum, L., Landva, A. (1966). "DIRECT SIMPLE-SHEAR TESTS ON A NORWEGIAN QUICK CLAY." Geotechnique, 16(1), 1-20.

Boulanger, R. W., Chan, C.K., Seed, H.B., Seed, R.B., Sousa, J.B. (1993). "A Low-

Compliance Bi-Directional Cyclic Simple Shear Apparatus." Geotechnical

Testing Journal, 16(1), 36-45.

Ishihara, K., Yamazaki, F. (1980). "Cyclic Simple Shear Tests on Saturated Sand in Multi-Directional Loading." Soils and Foundations, 20(1), 45-59.

Kammerer, A. M. (2002), Undrained Response of Monterey 0/30 Sand Under Multidirectional Cyclic Simple Shear Loading Conditions, Ph.D. Dissertation, University of California, Berkeley, 433 pp.

Roscoe, K. H. (1953). "An apparatus for the application of simple shear to soil samples." Proc., 3rd Int. Conf. Soil Mech. Foundation Engrg., London, 186-191

Saada, A. S., Fries, G., Ker, C. (1983). "An Evaluation of Laboratory Testing Techniques in Soil Mechanics." Soils and Foundations, 23(2), 98-112.

Wood, D. M., Drescher, A., and Budhu, M. (1980). "On the Determination of Stress State in Simple Shear Apparatus." Geotechnical Testing Journal, 2(4), 211-222. 